\title{
EXISTENCE, NONEXISTENCE AND MULTIPLICITY RESULTS FOR SEMILINEAR ELLIPTIC PROBLEMS WITH MEASURE DATA AND ABSORPTION-REACTION TERM
}

\author{
B. Abdellaoui, A. Primo, and T. M. Touaoula
}

Abstract. In the case where $g(u)$ appears as an absorption term, then under some additional hypotheses on $g$ we prove that the main problem has a solution for all $\lambda>0$ and for all positive $\mu \in L^{1}(\Omega)$. In the case where $g$ appears as a reaction term, then we prove that the main problem has at least two positive solutions under suitable hypotheses on $\mu$. The asymptotic linear case is also studied.

Mathematics subject classification (2000): 35D05, 35D10, 35J15, 35J20, 35J70.

Keywords and phrases: semilinear elliptic equations, existence and multiplicity, renormalized solutions, radon measures.

\section{REFERENCES}

[1] B. Abdellaoui, A. Dall'Aglio, I. Peral, Some Remarks on Elliptic Problems with Critical Growth in the Gradient, J. Differential Equations, 222 (2006), 21-62.

[2] B. Abdellaoui, E. Colorado, I. Peral, Some improved Caffarelli-Kohn-Nirenberg inequalities, Calc. Var. Partial Differential Equations, 23, 3 (2005), 327-345.

[3] B. Abdellaoui, E. Colorado, M. Sanchón, Regularity of entropy solutions of quasilinear elliptic problems related to Hardy-Sobolev inequalities, Adv. Nonlinear Stud., 6, 4 (2006), 547-562.

[4] Abdellaoui, B., Peral, I., On quasilinear elliptic equations related to some Caffarelli- KohnNirenberg inequalities, Commun. Pure Appl. Anal., 2, 4 (2003), 539-566.

[5] Abdellaoui, B., Peral, I., A note on a critical problem with natural growth in the gradient, J. Eur. Math. Soc., 8, 2 (2006), 157-170.

[6] A. Ambrosetti, G. Prodi, On the inversion of some differentiable mappings with singularities between Banach spaces, Ann. Mat. Pura Appl., 93, 4 (1972), 231-246.

[7] A. Ambrosetti, G. Prodi, A primer of nonlinear analysis, Cambridge Studies in Advanced Mathematics 34, Cambridge University Press, Cambridge, 1993.

[8] S. Alama, Semilinear elliptic equations with sublinear indefinite nonlinearities, Adv. Diff. Equations, 4, 6 (1999), 813-842.

[9] H. AMANN, P. QUITTNER, Elliptic boundary value problems involving measures: existence, regularity, and multiplicity, Adv. Differential Equations, 3, 6 (1998), 753-813.

[10] P. Baras, J.A. Goldstein, The heat equation with a singular potential, Trans. Amer. Math. Soc. 284, 1 (1984), 121-139.

[11] P. BARAS, M. PIERRE, Critre d'existence de solutions positives pour des quations semi-linaires non monotones, Ann. Inst. H. Poincar Anal. Non Linaire, 2, 3 (1985), 185-212.

[12] P. BARAS, M. Pierre, Singularits liminables pour des quations semi-linaires, Ann. Inst. Fourier, 34, 1 (1984), 185-206.

[13] P. Bnilan, H. Brezis, Nonlinear problems related to the Thomas-Fermi equation. Dedicated to Philippe Bnilan, J. Evol. Equ., 3, 4 (2003), 673-770.

[14] H. BERESTYCKI, Le nombre de solutions de certains problmes semi-linaires elliptiques, J. Funct. Anal., 40, 1 (1981), 1-29.

[15] L. BocCARdo, T. GallouËT, L. Orsina, Existence and nonexistence of solutions for some nonlinear elliptic equations, J. Anal. Math., 73 (1997), 203-223. 
[16] L. Boccardo, L. Orsina, I. Peral, A Remark on Existence and Optimal Summability of Solutions of Elliptic Problems Involving Hardy Potential, Discrete and Continuous Dynamical Systems, 16, 3 (2006), 513-523.

[17] H. Brezis, W.A. Strauss, Semilinear second order elliptic equations in $L^{1}$, J. Math. Soc. Japan, 25 (1973), 565-590.

[18] H. BreZIS, X. CABRÉ, Some simple nonlinear PDE's without solution, Boll. Unione. Mat. Ital. Sez. B, 8, 1 (1998), 223-262.

[19] H. Brezis, M. Marcus, A.C.Ponce, Nonlinear elliptic equations with measures revisited. Mathematical aspects of nonlinear dispersive equations, Ann. of Math. Stud., 163 (2007), 55-109.

[20] H. Brezis, L. Nirenberg, Positive solutions of nonlinear elliptic equations involving critical Sobolev exponents, Comm. Pure App. Math., Vol. XXXVI (1983), 437-477.

[21] Colorado, E., Peral, I., Eigenvalues and bifurcation for elliptic equations with mixed DirichletNeumann boundary conditions related to Caffarelli-Kohn-Nirenberg inequalities, Topol. Methods Nonlinear Anal., 23, 2 (2004), 239-273.

[22] G. Dal Maso, F. Murat, L. Orsina, A. Prignet, Renormalized solutions of elliptic equations with general measure data, Ann. Scuola Norm. Pisa CI. Sci., 28, 4 (1999), 741-808.

[23] P. Bénilan, L. Boccardo, T. Gallouët, R. Gariepy, M. Pierre, J.L. Vazquez, An $L^{1}$ Theory of Existence and Uniqueness of Solutions of Nonlinear Elliptic Equations, Ann. Scuola Norm. Sup. Pisa., 22, 2 (1995), 241-273.

[24] A. FerRero, C. SACCON, Existence and multiplicity results for semilinear equations with measure data, Topol. Methods Nonlinear Anal., 28, 2 (2006), 285-318.

[25] J. Garcia Azorero, I. Peral, Hardy inequalities and some critical elliptic and parabolic problems, J. Differential Equations, 144 (1998), 441-476.

[26] N. Ghoussoub, D. Preiss, A general mountain pass principle for locating and classifying critical points, Ann. Inst. Henri Poincaré Analyse non linéaire, Vol. 6, 5 (1989), 321-330.

[27] L. JEANJEAN, On the existence of bounded Palais-Smale sequences and application to a LandesmanLazer-type problem set on $R^{N}$, Proc. Roy. Soc. Edinburgh Sect. A, 129, 4 (1999), 787-809.

[28] T. KATO, Schrödinger operators with singular potentials, Israel J. Math., 13 (1972), 135-148.

[29] P. PUCCI, J. SERRIN, The strong maximum principle revisited, J. Differential Equations, 196, 1 (2004), $1-66$.

[30] M. STRUwe, Variational methods. Applications to nonlinear partial differential equations and Hamiltonian systems. Third edition, A Series of Modern Surveys in Mathematics, 34. Springer-Verlag, Berlin, 2000.

[31] Z. Q. WANG, M. WILlem, Caffarelli-Kohn-Nirenberg inequalities with remainder terms, J. Funct. Anal., 203, 2 (2003), 550-568. 